\title{
The value of transcranial Doppler imaging in children with tuberculous meningitis
}

\author{
Ronald van Toorn • H. Simon Schaaf • Regan Solomons • \\ Jacoba A. Laubscher • Johan F. Schoeman
}

Received: 23 April 2014 / Accepted: 2 May 2014 / Published online: 15 May 2014

(C) Springer-Verlag Berlin Heidelberg 2014

\begin{abstract}
Purpose Transcranial Doppler imaging (TCDI) is potentially a valuable investigational tool in children with tuberculous meningitis (TBM), a condition often complicated by pathology relevant to Doppler imaging such as raised intracranial pressure (ICP) and cerebral vasculopathies.

Methods Serial TCDI was performed on 20 TBM children with the aim of investigating cerebrovascular haemodynamics and the relationship between pulsatility index (PI) and ICP. Results We observed a poor correlation between ICP and PI in children with communicating hydrocephalus $(p=0.72)$. No decline in PI was noted following 7 days of medical therapy for communicating hydrocephalus $(p=0.78)$ despite a concomitant decline in ICP. Conversely, a decline in PI was noted in all four children with non-communicating hydrocephalus who underwent cerebrospinal fluid diversion. High blood flow velocities (BFV) in all the basal cerebral arteries were observed in 14 children (70 \%). The high BFV persisted for 7 days suggesting stenosis due to vasculitis rather than functional vasospasm. Complete middle cerebral artery (MCA) occlusion, subnormal mean MCA velocities $(<40 \mathrm{~cm} / \mathrm{s})$ and PIs $(<0.4)$ correlated with radiologically proven large cerebral infarcts.

Conclusions TCDI-derived PI is not a reliable indicator of raised ICP in children with tuberculous hydrocephalus. This may be attributed to individual variation of tuberculous vascular disease, possibly compromising cerebral vascular
\end{abstract}

R. van Toorn $(\bowtie) \cdot$ H. S. Schaaf $\cdot$ R. Solomons $\cdot$ J. F. Schoeman Department of Paediatrics and Child Health, Stellenbosch University and Tygerberg Children's Hospital, PO Box 19063, Tygerberg, Cape Town 7505, Western Cape, South Africa

e-mail: vtoorn@sun.ac.za

J. A. Laubscher

Biostatistics Unit, Medical Research Council of South Africa, Tygerberg, Cape Town, South Africa compliance and resistance. Basal artery stenosis secondary to vasculitis is observed during the acute stage of TBM in the majority of children.

Keywords Tuberculous meningitis · Transcranial Doppler · Pulsatility index $\cdot$ Intracranial pressure $\cdot$ Mean blood flow velocity

\section{Introduction}

Transcranial Doppler imaging (TCDI) is a safe, portable, noninvasive, inexpensive and non-ionizing method of assessing cerebral hydrodynamics and haemodynamics [1]. It can be repeated multiple times and used for serial monitoring. TCDI may provide accurate information on intracranial pressure (ICP) and cerebral blood flow velocities (BFV) which may help guide therapy in critically ill patients [1]. This applies especially to resource-poor countries where expensive monitoring equipment is often not available. However, controversy exists whether TCDI-derived pulsatility index (PI) is a reliable marker of raised ICP. Some studies report a linear relationship between the PI and measured ICP in hydrocephalic children, whilst others have failed to find such correlation [2-6].

To date, only one study examined the potential role of TCDI in tuberculous hydrocephalus [7]. Two groups of 15 children with tuberculous hydrocephalus and congenital hydrocephalus were prospectively examined by TCDI before and after cerebrospinal fluid (CSF) diversion. A clinically relevant fall in PI after CSF diversion only occurred in children with congenital hydrocephalus $(p=0.007)$. In tuberculous meningitis (TBM) patients with infarcts, there was no statistically significant drop in the PI pre- and post-shunt procedure.

TCDI also allows evaluation and monitoring of cerebral basal artery haemodynamics. Studies report an inverse 
relationship between ICP and cerebral blood flow (CBF) in young children with meningitis and hydrocephalus $[8,9]$. Ventricular peritoneal shunting permits a sudden increase in $\mathrm{CBF}$ and $\mathrm{BFV}$, and increases of above $30 \%$ have been reported [10]. Only one study investigated the use of TCDI in tuberculous cerebral vasculopathy [11]. This study found that TCDI allows distinction of three phases of arteriopathy (i.e. vasospasm, vasodilatation and stenosis). The study found a good correlation between each of the three stages with clinical findings (Glasgow coma score), radiological findings (ischemia on magnetic resonance imaging [MRI]) and outcome.

The present study sought to investigate the potential role of serial TCDI in the assessment of tuberculous hydrocephalus-related raised ICP and interventions aimed at reducing ICP, and the role of serial TCDI as a dynamic modality in the assessment of TBM-related vasculopathy and its possible predictive value in terms of the development of infarcts.

\section{Material and methods}

The study was conducted at Tygerberg Children's Hospital, a tertiary referral hospital in Cape Town, South Africa. The study population comprised 20 children less than 13 years of age who were admitted for TBM from June 2012 to June 2013.

A definite diagnosis of TBM was made when Mycobacterium tuberculosis was cultured and/or polymerase chain reaction (PCR)-positive from CSF. In all other cases, the diagnosis was "probable TBM" based on clinical signs of meningitis in the presence of characteristic CSF findings (macroscopically clear, pleocytosis usually with lymphocyte predominance, elevated protein and reduced glucose). In addition, two of the following criteria were required: other clinical specimens culturepositive for M. tuberculosis and/or positive TB histology, a positive tuberculin skin test, a chest radiograph compatible with $\mathrm{TB}$, a cranial computerized tomography (CT) or MRI compatible with TBM, growth failure with crossing of weight-for-age percentiles or finally, household contact with a sputum smear-positive pulmonary TB case.

The severity of disease was classified according to the refined Medical Research Council classification [12]. All children underwent contrasted CT imaging at admission. The degree of hydrocephalus was expressed as the Evans ratio (maximum ventricular width divided by the largest biparietal distance between the inner tables of the skull); a ratio of larger than 0.3 is indicative of hydrocephalus [13]. The site and size of all infarcts were recorded.
All children underwent short intensified anti-TB treatment (6 months of rifampicin, isoniazid, pyrazinamide and ethionamide [RHZEth] for HIV-uninfected and 9 months RHZEth for HIV-infected children. Children with non-communicating hydrocephalus, as determined by air encephalography, were referred for immediate ventriculoperitoneal shunting (VPS) or endoscopic third ventriculostomy (ETV). All children with communicating hydrocephalus were treated medically with acetazolamide $(50 \mathrm{mg} / \mathrm{kg} /$ day $)$ and furosemide $(1 \mathrm{mg} / \mathrm{kg} /$ day) during the first month of therapy to expedite normalization of ICP.

All TCDI studies were performed by one investigator (RVT) as a bedside investigation using a GE Healthcare Vivid S5 high-end Ultrasound machine (General Electric Medical Systems, Milwaukee, USA) with a 2-MHz probe. Recordings were performed on admission and repeated after 3 and 7 days. The transtemporal window was used to isonate the anterior cerebral artery (ACA), middle cerebral artery (MCA) and posterior cerebral artery (PCA). The main horizontal section of the MCA from its origin to the limen insulae was isonated. This M1 segment of the MCA gives rise to numerous, small lenticostriate or perforating branches which are most commonly affected in TBM. Different depth range, flow direction and normal age-related flow velocity ranges have been established for each vessel [14]. The vessels were identified using these parameters.

Recorded parameters included peak, mean and diastolic flow velocities and PI. The PI is calculated by the Gosling index $(\mathrm{PI}=$ peak systolic velocity-end diastolic velocity) divided by the mean velocity. The normal range of PI in the MCA is $0.5-1.1$ [14]. Studies evaluating the variability in normal individuals found the PI to vary between 0.69 and 0.71 for the MCA, ACA and PCA, and there were no significant $(>20 \%)$ side-to-side or day-to-day differences [14]. The PI is automatically calculated and displayed on the TCDI instrument. Sample volumes were taken at a standardized distance from the Circle of Willis in all vessels bilaterally to allow for meaningful interpretation on follow-up examinations. At least three readings per vessel were made at each evaluation and the average measurement was used. Indices were determined using at least three cardiac cycles of visually and audibly good quality. In children with focal neurological deficits or radiological proven infarcts, PI measurements from the unaffected side (cerebral hemisphere) were used.

The peak systolic velocity was used to define the presence of stenosis. A peak systolic velocity of less than $140 \mathrm{~cm} / \mathrm{s}$ was considered to indicate a normal artery [14]. The degree of stenosis was qualified according to the published criteria and was graded into three categories: grade I $(140-209 \mathrm{~cm} / \mathrm{s})$, grade II $(210$ to $280 \mathrm{~cm} / \mathrm{s})$ and grade III $(>280 \mathrm{~cm} / \mathrm{s})$. MCA occlusion was diagnosed if all the basal arteries except the MCA in question were detectable or if the asymmetry index of the symptomatic MCA was $<21 \%$ compared with that of the 
contralateral MCA. The Lindegaard index (the ratio of the MCA velocity to the extracranial internal carotid artery velocity) was used to distinguish cerebral vasospasm from hyperemia [14]. Vasospasm was diagnosed in cases where the mean MCA flow velocity exceeded $120 \mathrm{~cm} / \mathrm{s}$, and the Lindegaard ratio was more than 3 .

TCDI measurements may be influenced by different physiological and pathological factors such as age, gender, hematocrit, fever, hypoglycemia, heart rate, blood pressure and drugs with either vasodilatation or vasoconstriction properties. All physiological parameters were kept stable during the TCDI examination. Carbon dioxide $\left(\mathrm{PaCO}_{2}\right)$ levels were not measured as angiographic studies have demonstrated that the larger human basal cerebral arteries are unresponsive (at least within the tolerances of measurements) to changes in $\mathrm{PaCO}_{2}$, while arteries that had diameters less than $1 \mathrm{~mm}$ responded [15]. None of the TBM children were ventilated or exhibited abnormal breathing patterns during the TCDI.

Lumbar CSF pressure was recorded immediately after TCDI upon admission, after 3 and 7 days. The opening pressure measurement was determined using the highest resting $\mathrm{CSF}$ level, in centimetres $\mathrm{H}_{2} \mathrm{O}$, when sustained for at least $10 \mathrm{~s}$. A CSF baseline pressure of greater than $20 \mathrm{~cm} \mathrm{H}_{2} \mathrm{O}$ was considered indicative of raised ICP in children with communicating hydrocephalus.

Neurological outcome at 6 months was divided into the following four categories: (1) normal, including normal motor function, intelligence, vision and hearing; (2) mild sequelae, including hemiparesis, mild intellectual impairment and impaired vision and/or hearing; (3) severe sequelae, including quadriparesis, severe intellectual impairment, blindness and/ or deafness; and (4) death. Clinical outcome was defined as "good" in the case of normal and mild neurological sequelae and "poor" in children with severe neurological sequelae or death.

The study was approved by the Health Research Ethics Committee of Stellenbosch University (N12/07/041).

\section{Results}

Of the 20 children with TBM, median age of 37 months (range 4-140 months), 12 (60\%) were male. Sixteen ( $80 \%$ ) of the children presented with stage II TBM, three (15\%) with stage III TBM and one (5\%) had stage I TBM; three (15\%) were HIV-infected. In seven children (35\%), M. tuberculosis was isolated from gastric aspirates $(n=6)$ or CSF $(n=1)$; six isolates proved drug-susceptible and one was isoniazidmonoresistant.

Sixteen $(80 \%)$ children had communicating hydrocephalus; in the four $(20 \%)$ children with non-communicating hydrocephalus, three underwent VPS and one ETV. Ten of the $16(62 \%)$ children with communicating hydrocephalus had elevated ICP on admission (median ICP $35 \mathrm{~cm} \mathrm{H}_{2} \mathrm{O}$, range $22-55)$. Eight (40 \%) had visible infarcts on admission cranial CT imaging. No correlation was found between the ventricular size (Evans' index) on CT and ICP in children with communicating hydrocephalus ( $p=0.73$ ).

Figure 1 illustrates the poor correlation between serial ICP and PI (43 measurements) in the 16 children with communicating tuberculous hydrocephalus $(p=0.72)$. Cases with confirmed non-communicating hydrocephalus were not included in this correlation because lumbar CSF pressure does not necessarily reflect ICP in this condition. Figure 2 illustrates the change in ICP on days 1, 3 and 7 in the 16 children who underwent medical treatment for communicating hydrocephalus. In all cases, the ICP normalized within 7 days after initiation of acetazolamide and furosemide. In contrast, there was no significant decline in the PI after 7 days of medical therapy $(p=0.78)$. However, in all four children with noncommunicating hydrocephalus, the PI decreased following CSF diversion (Fig. 3).

Bilateral MCA stenosis on admission was detected in 14 (70\%) of the 20 TBM children; 10 had grade I stenosis and 4 had grade II stenosis (Fig. 4). Serial TCDI on days 1, 3 and 7 showed no change in the grade of stenosis in 11 children, improvement in 2 and deterioration in 1 . One child initially presented with a grade II MCA stenosis (Lindegard index $>3$ ) which normalized on day 3 and remained normal on day 7 . This indicates vasospasm. There was no significant difference in peak systolic flow velocities on day 1 compared to day 7 in the 20 TBM patients $(p=0.80)$. Similarly, there was no significant difference in the mean MCA BFV on day 1 compared to day 7 in the 16 children with communicating hydrocephalus $(p=0.18)$.

Side-to-side differences in MCA flow velocities were detected in four of the eight children with radiologically proven infarcts. In two of the children, subnormal mean MCA velocities $(\leq 40 \mathrm{~cm} / \mathrm{s})$ and PI $(\leq 0.4)$ resulted in ipsilateral infarction. Complete MCA occlusion (all other basal arteries detectable except the MCA in question) occurred in the other two children; both had large MCA infarcts on CT and severe hemiplegias.

Eighteen $(90 \%)$ of the 20 children had a good outcome after 6 months of treatment. The two children with poor outcome both had stage III disease, infarcts on CT and subnormal and declining PI and mean MCA velocities on TCDI. All children with a good outcome had normal TCDI.

\section{Discussion}

For optimal management of acute TBM in children, ICP should be measured in order to determine its contribution to the patient's neurological state (e.g. coma) and because it is treatable. Studies have shown that clinical diagnosis of the 
Fig. 1 Fit plot for intracranial pressure (ICP). Illustration of the poor correlation between ICP and pulsatility index in the 16 children with communicating hydrocephalus on days 1,3 and 7

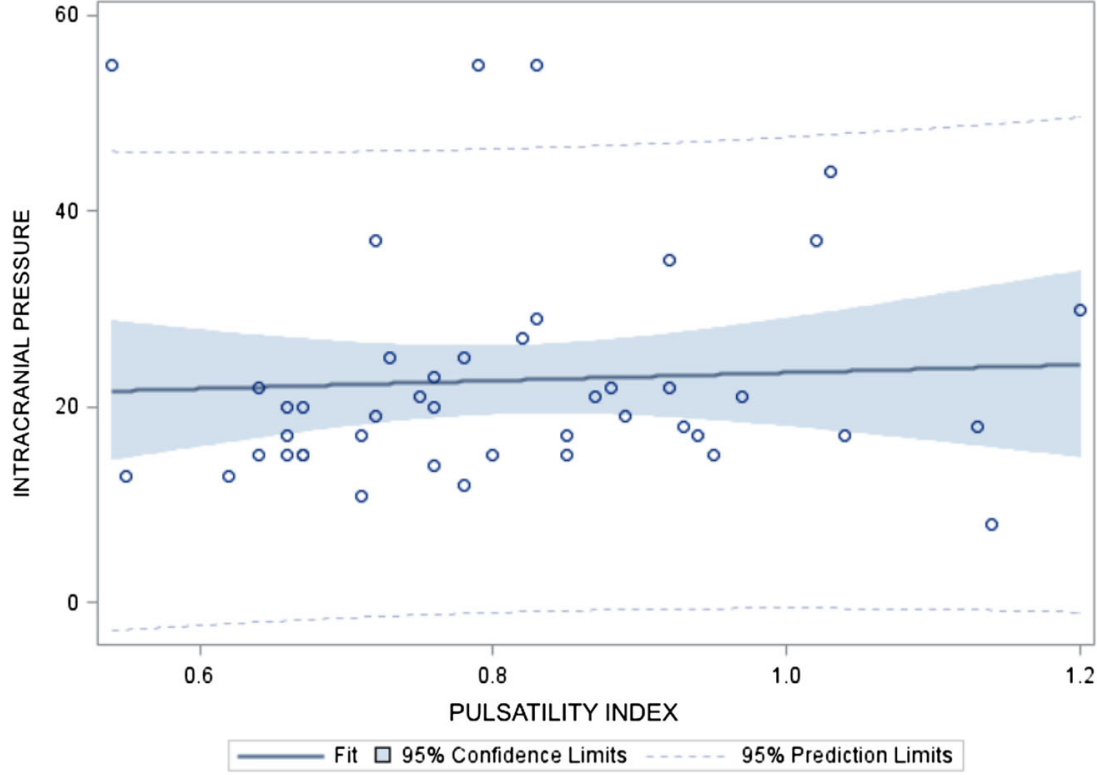

presence and degree of raised ICP is unreliable, especially in children with closed anterior fontanels [16]. The value of CT imaging is limited by the poor correlation between the degree of hydrocephalus (ventricular size) and ICP [16]. Similarly, in this study, no correlation was found between ventricular size on CT and the degree of raised ICP ( $p=0.73)$.

Many non-invasive techniques of measuring ICP have been evaluated, such as intraocular pressure, tympanic membrane displacement and measurement of intracranial blood flow [17]. None has been found reliable enough to replace invasive procedures. Lumbar CSF pressure measurement in TBM accurately reflects ICP in children with communicating tuberculous hydrocephalus. In this condition, it can be repeatedly done to monitor the course without any danger to the patient; repeated lumbar puncture has even been advocated as a safe although invasive treatment option for communicating hydrocephalus in TBM [18]. In contrast, lumbar CSF pressure does not reflect ICP in non-communicating tuberculous hydrocephalus; in this condition, a lumbar puncture may result in cerebral transtentorial or uncal herniation with dire consequences for the patient. A non-invasive method of measuring ICP in children with TBM would therefore potentially be of great value, as it may assist with identification of hydrocephalic children who require CSF drainage procedures, assist with assessment of shunt function, confirm fenestration patency in patients with non-communicating tuberculous hydrocephalus who underwent ETV and assist with evaluating the therapeutic effects of ICP-lowering drugs such as mannitol and diuretics.
Fig. 2 Illustration of the ICP on days 1,3 and 7 in 16 TBM children with communicating hydrocephalus following medical therapy consisting of acetazolamide and furosemide
Intracranial pressure per patient on Day 1,3 \& 7

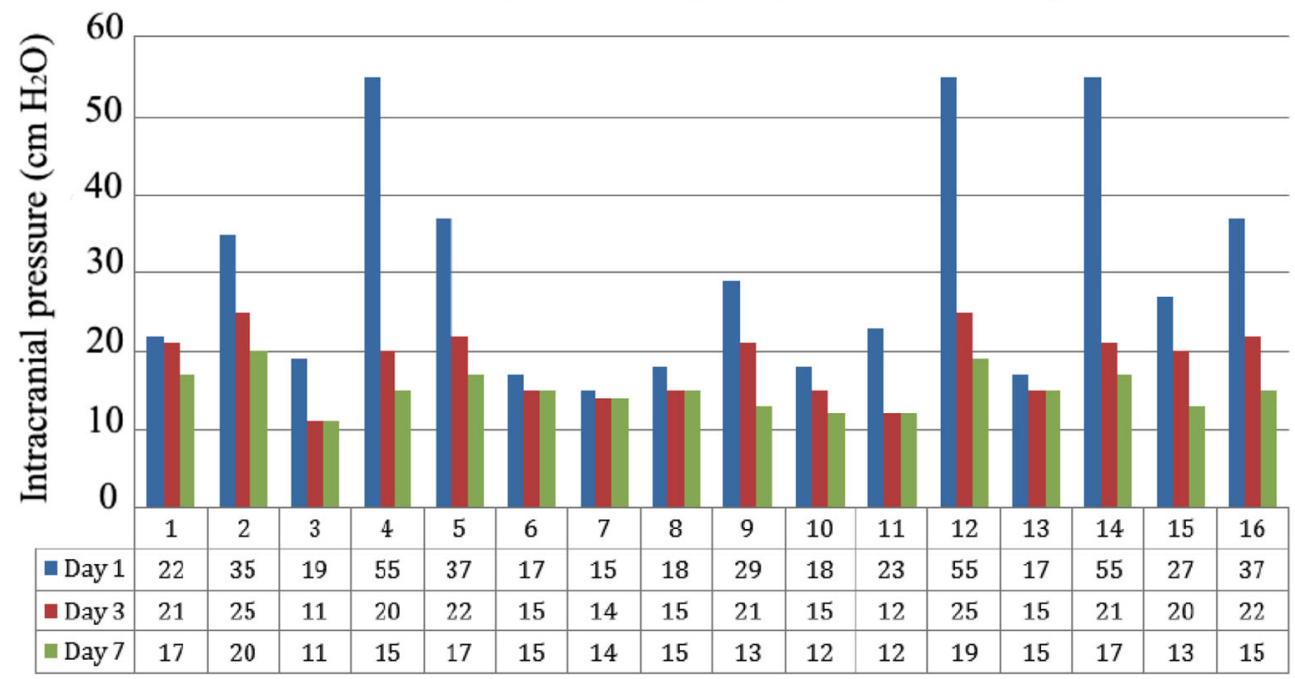


Fig. 3 Illustration of the change in pulsatility index before and after cerebrospinal fluid diversion in four tuberculous meningitis children with non-communicating hydrocephalus. Patients 1-3 underwent ventriculoperitoneal shunting and patient 4 underwent endoscopic third ventriculostomy

\section{Pulsatility Index (PI) following CSF diversion}

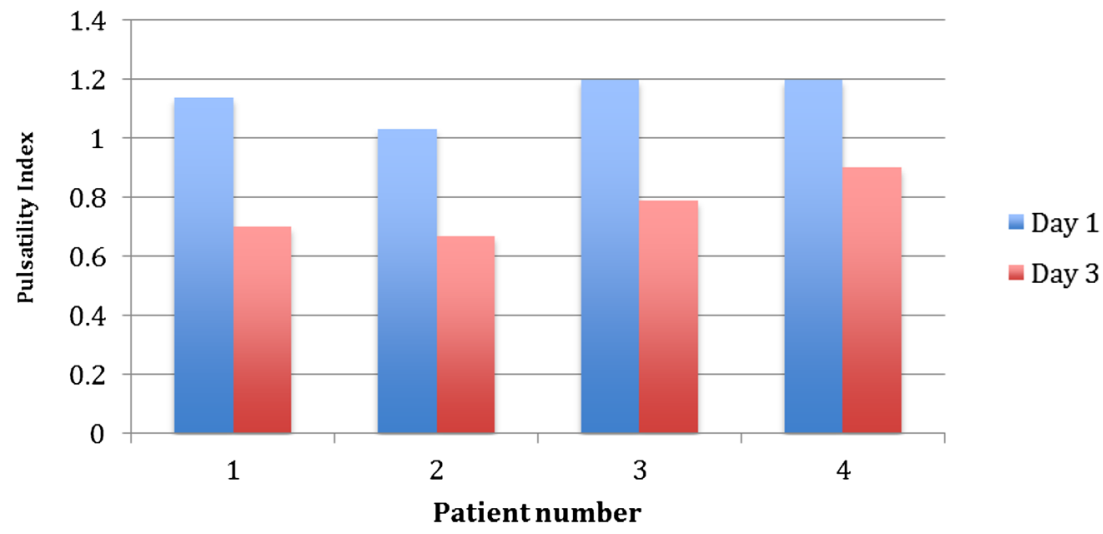

In our study, TCDI-related PI was found not to be an accurate method of assessing ICP in children with communicating tuberculous hydrocephalus. No linear correlation was found between PI and ICP $(p=0.72)$. This may be attributed to individual variation of tuberculous vascular disease, possibly compromising cerebral vascular compliance and resistance. It has been suggested that absolute PI values should be read with extreme caution, even at the extremes of ICP values, and that TCDI can play an important role as a means of monitoring pressure changes over time [3]. In our study, no decline in PI was noted following 7 days of medical therapy for communicating hydrocephalus, despite a confirmed decline in ICP. Conversely, a decline in PI was noted in all four children with non-communicating hydrocephalus who underwent CSF diversion, most likely as a result of a more dramatic fall in ICP in this group (Fig. 3). Larger studies are warranted to investigate this finding and should ideally include pre- and postsurgical ICP monitoring.

Carbonic anhydrase inhibitors (acetazolamide) and loop diuretics (furosemide) exert their effect on ICP by reducing CSF production at the choroid plexus [19]. Acetazolamide has a rapid onset of action and has been found to reduce CSF production in humans by $6-50 \%$ [20]. Inhibition of CSF flow occurs once $99.5 \%$ of choroid plexus carbonic anhydrase is inhibited [21]. In children with communicating tuberculous hydrocephalus, acetazolamide and furosemide in combination have been found to be more effective in achieving normal ICP than antituberculous drugs alone [16]. In this study, rapid normalization of ICP occurred within 7 days of therapy in all 10 cases of communicating tuberculous hydrocephalus who had elevated ICP (Fig. 2). Acetazolamide has also been shown not to alter the cerebral metabolic rate of oxygen, which is beneficial in children with TBM-related ischemic brain disease [22].

Acetazolamide also increases CBF by $5-80 \%$ [22]. High bilateral MCA BFV upon admission was observed in $70 \%$ of the TBM children. This occurred prior to acetazolamide administration and CSF sampling and can be ascribed to vessel narrowing (or stretching on account of acute hydrocephalus (ventricular dilatation) or spasm because of meningeal involvement (as in acute subarachnoid haemorrhage)). In almost all the children, high BFV persisted after 1 week which supports vessel narrowing secondary to organic stenosis rather than functional vasospasm. Functional vasospasm was observed in only one child. In this child, no infarcts were observed on CT and outcome after 6 months was normal.

Information regarding the sensitivity and specificity of TCDI in the diagnosis of cerebral ischemia in general is limited [23]. In this study, subnormal mean MCA BFV $(\leq 40 \mathrm{~cm} / \mathrm{s})$ and PI $(\leq 0.4)$ were associated with CT infarction in two children. The diminished MCA BFV reflects decreased perfusion (demand) to the infarcted area. Tuberculous vasculopathy of the distal internal carotid artery may also account for the diminished MCA BFV. In four children with territory infarcts on admission, no side-to-side differences in MCA BFV or subnormal PIs were detected. This can be attributed to the occlusion of a limited number (one or two) of the nine MCA perforators which has been shown not to affect the haemodynamics of the MCA [23]. This could unfortunately not be confirmed due to lack of concomitant MR angiography.

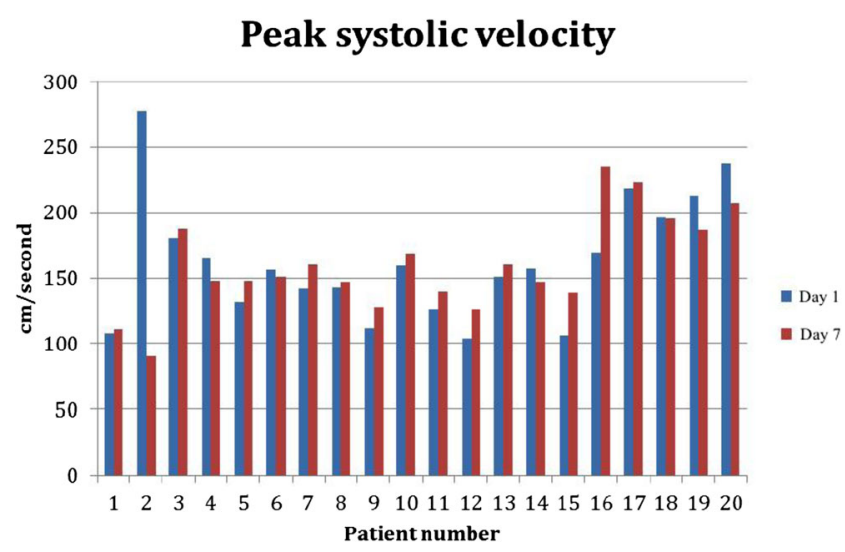

Fig. 4 Illustration of the peak middle cerebral artery systolic blood flow velocity on admission compared to day 7 in 20 children with TBM 
Acknowledgments The study received financial support from the National Research Foundation-HS Schaaf.

Conflict of interest None

\section{References}

1. Kassab MY, Majid A, Farooq M, Azhary H, Hershey LA, Bednarczyk EM, Graybeal DF, Johnson MD (2007) Transcranial Doppler: an introduction for primary care physicians. J Am Board Fam Med 20:65-71

2. Bellner J, Rommer B, Reinstrup P, Kristiansson KA, Ryding E, Brandt L (2004) Transcranial Doppler sonography pulsatility index (PI) reflects intracranial pressure (ICP). Surg Neurol 62:45-51

3. Wakerley BR, Sharma VK (2013) Transcranial Doppler derived pulsatility index in the assessment of intracranial pressure: the trend is your friend. Neurosurgery 72(2):E319-E320

4. Hanlo PW, Gooskens RH, Nijhuis IJ, Faber JA, Peters RJ, van Huffelen AC, Tulleken CA, Willemse J (1995) Value of transcranial Doppler indices in predicting raised ICP in infantile hydrocephalus. A study with review of the literature. Childs Nerv Syst 11(10):595603

5. Behrens A, Lenfeldt N, Ambarki K, Malm J, Eklund A, Koskinen LO (2011) Intracranial pressure and pulsatility index. Neurosurgery 69(4):E1033-E1034

6. Figaji AA, Zwane E, Fieggen AG, Siesjo P, Peter JC (2009) Transcranial Doppler pulsatility index is not a reliable indicator of intracranial pressure in children with severe traumatic brain injury. Surg Neurol 4:389-394

7. Nadvi SS, Du Trevou MD, Van Dellen JR, Gouws E (1994) The use of transcranial Doppler ultrasonography as a method of assessing intracranial pressure in hydrocephalic children. Br J Neurosurg 8(5): 573-577

8. Goh D, Minns RA (1993) Cerebral blood flow velocity monitoring in pyogenic meningitis. Arch Dis Child 68(1):111-119

9. McMenamin JB, Volpe JJ (1984) Bacterial meningitis in infancy: effects on intracranial pressure and cerebral blood flow velocity. Neurology 34(4):500-504

10. Pople IK, Quinn MW, Bayson R, Hayward RD (1991) The Doppler pulsitility index as a screening test for blocked ventriculo-peritoneal shunts. Eur J Pediatr Surg 1(1 Suppl):27-29
11. Kilic T, Elmaci I, Ozek MM, Pamir MN (2002) Utility of transcranial Doppler ultrasonography in the diagnosis and follow-up of tuberculous meningitis-related vasculopathy. Childs Nerv Syst 18(3-4):142146

12. van Toorn R, Springer P, Laubscher JA, Schoeman JF (2012) Value of different staging systems for predicting neurological outcome in children with tuberculous meningitis. Int J Tuberc Lung Dis 16(5): $628-632$

13. von Benzing H, Andronikou S, van Toorn R, Douglas T (2012) Are axial measurements and computerized volumetric ratios determined from axial MRI useful for diagnosing hydrocephalus in children with tuberculous meningitis? Childs Nerv Syst 28(1):79-85

14. Arnolds BJ, von Reutern GM (1986) Transcranial Doppler sonography. Examination technique and normal reference values. Ultrasound Med Biol 12(2):115-123

15. Huber P, Handa J (1967) Effect of contrast material, hypercapnia, hyperventilation, hypertonic saline and papaverine on the diameter of the cerebral arteries. Invest Radiol 2(1):17-32

16. Schoeman J, Donald P, van Zyl L, Keet M, Wait J (1991) Tuberculous hydrocephalus: comparison of different treatment with regard to ICP, ventricular size and clinical outcome. Dev Med Child Neuro 33(5):396-405

17. Rosenberg JB, Shiloh AL, Savel RH, Eisen LA (2011) Non-invasive methods of estimating intracranial pressure. Neurocrit Care 15(3): 599-608

18. Visudhiphan P, Chiemchanya S (1979) Hydrocephalus in tuberculous meningitis in children: treatment with acetazolamide and repeated lumbar puncture. J Pediatr 95(4):657-660

19. Carrion E, Hertzog JH, Medlock MD, Hauser GJ, Dalton HJ (2001) Use of acetazolamide to decrease cerebrospinal fluid production in chronically ventilated patients with ventriculoperitoneal shunts. Arch Dis Child 84(1):68-71

20. Rubin RC, Henderson ES, Ommaya AK, Walker MD, Rall DP (1966) The production of cerebrospinal fluid in man and its modification by acetazolamide. J Neurosurg 25(4):430-436

21. McCarthy KD, Reed DJ (1974) The effect of acetazolamide and furosemide on CSF production and carbonic anhydrase activity. J Pharmacol Exp Ther 189:194-201

22. Vorstrup S, Hendriksen L, Paulson OB (1984) Effect of acetazolamide on cerebral blood flow and cerebral metabolic rate of oxygen. J Clin Invest 74(5):1634-1639

23. Zanette EM, Fieschi C, Bozzao L, Roberti C, Toni D, Argentino C, Lenzi GL (1989) Comparison of cerebral angiography and transcranial Doppler sonography in acute stroke. Stroke 20(7):899-903 